\title{
Fe(III) distribution varies substantially within and between atherosclerotic plaques
}

\author{
Håkan Gustafsson, Martin Hallbeck, M. Norell, Mikael Lindgren, Maria Engström, Anders \\ Rosén and Helene Zachrisson
}

\section{Linköping University Post Print}

\section{Tweet}

N.B.: When citing this work, cite the original article.

Original Publication:

Håkan Gustafsson, Martin Hallbeck, M. Norell, Mikael Lindgren, Maria Engström, Anders Rosén and Helene Zachrisson, Fe(III) distribution varies substantially within and between atherosclerotic plaques, 2014, Magnetic Resonance in Medicine, (2), 71, 885-892.

http://dx.doi.org/10.1002/mrm.24687

Copyright: Wiley http://eu.wiley.com/WileyCDA/

Postprint available at: Linköping University Electronic Press http://urn.kb.se/resolve?urn=urn:nbn:se:liu:diva-98001 
Fe(III) distribution varies substantially within and between atherosclerotic plaques

H. Gustafsson* (1,2), M . Hallbeck (3), M. Norell (4), M. Lindgren (5), M. Engström (1,2), A. Rosén (6) and H. Zachrisson (4).

1. Department of Medical and Health Sciences (IMH), Division of Radiological Sciences, Linköping University.

2. Center for Medical Image Science and Visualization (CMIV), Linköping University.

3. Pathology, Faculty of Health Sciences, Department of Clinical and Experimental Medicine, Linköping University, Department of Clinical Pathology, County Council of Östergötland, Linköping, Sweden.

4. Department of Medical and Health Sciences, Division of Clinical Physiology, Linköping University.

5. Department of Physics, Norwegian University of Science and Technology, 7491 Trondheim, Norway.

6. Department of Clinical and Experimental Medicine (IKE), Division of Cell Biology, Linköping University.

* Corresponding author. Contact details:

E-mail: hakan.l.gustafsson@liu.se 
Adress: Håkan Gustafsson, IMH/Radiation Physics, Linköping University, 58185 Linköping, Sweden

Telephone: +46 (0)101031475 or +46(0)701902113

Fax: Not available

Word count of body (not including the title page, abstract, figure captions, tables, table captions, or references): 4108 words.

Word count of abstract: 199 words.

Total number of figures and tables: 5 figures and 3 tables. 
Objective: Vulnerable atherosclerotic plaques are structurally weak and prone to rupture, presumably due to local oxidative stress. Redox active iron is linked to oxidative stress and the aim of this study was to investigate the distribution of $\mathrm{Fe}(\mathrm{III})$ in carotid plaques and its relation to vulnerability for rupture.

Methods: Atherosclerotic plaques from ten patients (3 asymptomatic and 7 symptomatic) were investigated. Plaque vulnerability was classified using ultrasound and immunohistochemistry and correlated to $\mathrm{Fe}(\mathrm{III})$ measured by electron paramagnetic resonance spectroscopy.

Results: Large intra-plaque Fe(III) variations were found. Plaques from symptomatic patients had a higher Fe(III) concentration as compared to asymptomatic plaques $(0.36 \pm 0.21$ vs. 0.06 $\pm 0.04 \mathrm{nmol} \mathrm{Fe}(\mathrm{III}) / \mathrm{mg}$ tissue, $\mathrm{P}<0.05$, in sections adjoining narrowest part of the plaques). All but one plaque from symptomatic patients showed signs of cap rupture. No plaque from asymptomatic patients showed signs of cap rupture. There was a significant increase in cap macrophages in plaques from symptomatic patients compared to asymptomatic patients $(31 \pm$ $11 \%$ vs. $2.3 \pm 2.3 \%, \mathrm{P}<0.01)$.

Conclusion: Fe(III) distribution varies substantially within atherosclerotic plaques. Plaques from symptomatic patients had significantly higher concentrations of Fe(III), signs of cap rupture and increased cap macrophage activity.

\section{Keywords}

Atherosclerosis, oxidative stress, reactive oxygen species, iron, electron paramagnetic resonance (EPR). 


\section{Introduction}

Atherosclerotic plaques vulnerable for rupture are characterised by a thin fibrous cap [1], a large lipid pool and a high number of inflammatory cells such as macrophages-derived foam cells [2-4]. Vulnerable plaques are also structurally weaker [5] and therefore more likely to rupture in response to physical forces [6]. Oxidative stress, i.e. imbalance between physiological oxidative defence mechanisms and reactive oxygen species (ROS), causes biological damage [7-9]. A weak fibrous cap in vulnerable plaques may be a result of oxidative damage by high local concentrations of ROS [9] produced by macrophage derived foam cells. It has also been demonstrated that high local concentration of ROS leads to increased activity of vascular matrix metalloproteinase (MMP) that further degrades the fibrous cap $[6,10]$.

Atherosclerotic plaques in the carotid arteries is a common source of thromboembolism and subsequent risk of stroke. Carotid endarterectomy (CEA) guidelines in symptomatic carotid artery stenosis are based on European Carotid Surgery Trial (ECST) and North American Symptomatic Carotid Endarterectomy Trial (NASCET) criteria with 70\% or greater stenosis [11]. Also asymptomatic patients with a high grade internal carotid artery stenosis may benefit from carotid endarterectomy as demonstrated in the asymptomatic carotid atherosclerosis study (ACAS) [12] and the asymptomatic carotid surgery trial (ACST) [13]. Although ultrasonic duplex imaging of the carotid artery is routinely used in the clinic to assess the level of stenosis and blood flow, the value of ultrasonic duplex imaging for assessment of plaque composition and plaque vulnerability for rupture remains limited [14]. Previous results indicate that the ultrasound technique could provide useful information both for the evaluation of patient risk and for guidance of therapeutic decisions and monitoring. Contradictory results, however, make further investigations necessary. Some of these are 
aimed at the description of plaque surface (the cap) and the subcapsular area with possible necrotic zones including macrophage activity.

A number of studies suggest that iron may play a key role in the pathogenesis of atherosclerosis [15-23]. Redox-active iron is known to act as a catalyst in the production of strong oxidative ROS (Fenton and Haber-Weiss reactions) [24-25] and it has been hypothesised [20] that elevated levels of iron in vivo lead to increased oxidative stress. Recently Raman et al. [19] used magnetic resonance imaging (MRI) to quantify iron content in carotid atherosclerotic plaques in vivo in human patients using standard methods for MRI tissue iron assessment [26-28] and showed that MRI can be used to distinguish symptom producing plaques from asymptomatic plaques. It is of interest to further study the relationship between iron levels in atherosclerotic plaques and vulnerability for rupture, especially since it has already been established that MRI can be used for assessment of other risk factors for rupture such as microemboli, cap-rupture, intra-plaque haemorrhage, plaque neovasculature and inflammation [29-30].

Various techniques have been used to study iron in biological systems [31]. Electron paramagnetic resonance (EPR) spectroscopy (equivalent to electron spin resonance (ESR) spectroscopy) offers a non-destructive method for studies of paramagnetic iron species to obtain information on e.g., the iron oxidation state and the type of iron-ligand bonding [32]. EPR spectroscopy can in addition be used for absolute quantifications of high spin ferric $\mathrm{Fe}(\mathrm{III})$ ions in frozen samples in the micro-molar range [31]. The EPR spectra of frozen human carotid atherosclerotic plaques consist to various degrees of signals assigned to haem iron, non-haem high-spin iron (e.g. iron bond in transferrin) and low-spin iron [16-17, 19, 3233]. Although some states of Fe(II) are paramagnetic, an EPR signal is not typically observed 
for $\mathrm{Fe}(\mathrm{II})$ due to very strong spin-orbit coupling [32-33] giving rise to substantial linebroadening.

The high reactivity of some of the ROS (e.g. hydroxyl radicals) limits the distance between the chemical origin and the point for oxidative damage. We hypothesized that vulnerability for rupture of carotid atherosclerotic plaques is better correlated to intra-plaque iron distribution and therefore to the localization of oxidative stress, rather than to a high mean iron concentration in the plaque. In the present work we therefore quantified the distribution of iron both in plaques from symptomatic and asymptomatic patients with the aim of exploring the relationship between elevated local levels of redox active iron and plaque vulnerability for rupture as classified by in vivo and ex vivo ultrasound and compared to histopathological examination.

\section{Materials and methods}

\section{Patient data}

Ten patients undergoing carotid endarterectomy (CEA) were prospectively and consecutively enrolled for study participation. Seven patients had symptomatic ICA (internal carotid artery) stenosis (amauroisis fugax, $\mathrm{n}=2$; minor stroke $\mathrm{n}=4$; transient ischaemic attack (TIA), $\mathrm{n}=1$ ) and 3 patients were asymptomatic. The symptomatic patient group was represented by 5 males (mean age 75 years (y); range 60-83 y) and 2 women (mean age 58 y; range 53-63 y). The asymptomatic patient group was represented by 2 males (mean age 69 y; range 55-83 y) and one woman (age 68 y). The study was approved by the Local Ethical Review Board in Linköping, Sweden. The plaques were surgically removed and immediately fixed in $40 \%$ formaldehyde. 


\section{Duplex ultrasound in vivo}

Preoperative duplex ultrasound of the neck vessels was performed within one week before carotid endarterectomy (CEA) in the seven symptomatic patients and one month before CEA in the three asymptomatic patients. Ultrasound B-mode imaging of the neck vessels was performed by a high frequency ultrasound Acuson S2000 scanner (Siemens, Mountain View, CA) equipped with a $9 \mathrm{MHz}-18 \mathrm{MHz}$ transducer. All conventional ultrasound measurements were performed at an angle of 60 degrees at maximum between the transducer and the blood flow direction and saved for further offline analysis using computerized image analysis software; Adobe Photoshop CS5 (Adobe systems, Mountain View, CA) The degree of ICA stenosis was measured by peak systolic flow velocities (PSV) to assess the degree of stenosis (ECST method) [34]. Plaque composition was objectively assigned as fibrous, fibroatheromatous or atheromatous and the caps were classified as smooth, irregular, or ulcerated.

\section{Duplex ultrasound ex vivo}

All ten carotid plaques were moulded into gelatine in special Plexiglas boxes $(15 \mathrm{~cm} \times 8 \mathrm{~cm}$ $\times 5 \mathrm{~cm}$ ) directly after CEA to allow for ex vivo ultrasound examination and subsequent quantification of $\mathrm{Fe}(\mathrm{III})$ using EPR spectroscopy and immunohistology. The moulding was performed in two steps: First $18.2 \mathrm{~g}$ of gelatine leafs (gelatine from animal origin, Dr. Oetker, Mölndal, Sweden) were dissolved in $3.5 \mathrm{dl}$ water in a beaker using a magnetic stirrer/heater until completely dissolved. The solution was then cooled down to $37^{\circ} \mathrm{C}$ by placing the beaker in a water bath and then poured into the Plexiglas box to a height of $3 \mathrm{~cm}$. The gelatine was then allowed to solidify by placing the Plexiglas box into a refrigerator $\left(8^{\circ} \mathrm{C}\right)$. After the gelatine had solidified a top layer of gelatine solution was prepared as described above and poured into the Plexiglas box to fill it up completely. Using a pair of tweezers the carotid plaque was placed at the bottom of the top layer of gelatine $(2 \mathrm{~cm}$ from the surface of 
the top layer). Care was taken to remove all air bubbles from the surface of the plaque, within the lumen of the plaque, at the surface of the bottom layer and at the surface of the top layer of gelatine. The top layer with plaque was then allowed to solidify by placing the box into the refrigerator again. Ultrasound B-mode imaging of the ex vivo plaques were performed using the same equipment as used for in vivo ultrasound. All carotid specimens underwent ultrasound examinations in both $2 \mathrm{D}$ and $3 \mathrm{D}$ to carefully determine the positions of the most constricted part of the plaque lumen which were documented with marks on a photograph of the plaque in its box.

\section{Histopathology}

From each of the ten plaques a $4 \mathrm{~mm}$ thick slice from the most constricted part of the vessel as determined by ultrasound was removed. This section was formalin fixed and paraffin embedded using standard methods for subsequent histopathological evaluation. The plaque parts distal and proximal to this centre slice were stored for subsequent quantification of Fe(III) using EPR spectroscopy (described below). For histopathology $4 \mu \mathrm{m}$ thick sections were sectioned. One of these was used for standard hematoxylin and eosine staining (H\&E). Subsequent sections were used for immunohistochemical staining with anti-CD68 antibody (Ab) (PG-M1, Dako, 1:100), anti-CD163 Ab (10D6, Leica Novocastra, 1:100), anti-CD31 Ab (JC/70A, Dako, 1:50) and anti-SMA Ab (1A4, Dako, 1:300). Staining was performed by using IntelliPATH FLX (BioCare). All stained sections were subsequently digitalized (ScanScope AT Turbo, Aperio). All cases were analysed by an experienced pathologist (M.H.). Analysis of the digitalized sections was performed with ImageScope (Aperio), the $H \& E$ section were used as the basis for analysis from which cap thickness and the presence of cap rupture could be analysed. The percentage of foamy macrophages within the cap was calculated by measuring the areas containing lipid laden macrophages (asserted on H\&E, 
confirmed by positive CD68 and CD163 staining) and dividing this with the total area of the cap. Area measurements were done using the ImageScope. Presence of rupture was evaluated on the H\&E section as was measurements of cap thickness, diameter of the lipid core and presence of calcification. Arbitrary values were given to the presence of macrophages outside of the plaque, microvascular proliferation in the cap (determined by CD31 and SMA labelling) and in other parts of the slide, cap thickness and hemosiderin content and presence of calcification. The observations from each slide were assigned numerical values based on the relative prominence: none $=0 ;$ minimal $=0-1$; moderate $=1-2$; extensive $=2-3$. In some cases small parts of the section were damaged during sectioning procedure due to calcifications, but in the sections used, the damage was not significant enough to preclude evaluation. Two of the symptomatic case was too damaged to permit histopathological evaluation and had to be excluded from this part of the study.

The plaque composition was classified as atheromatous, fibroatheromathous or fibrous. Presence of cap rupture was classified as "yes" or "no".

\section{Quantification of Fe(III)}

All EPR measurements were performed using a Bruker E5oo Elexsys X-Band EPR spectrometer equipped with a SHQE resonator at $150 \mathrm{~K}$ using a Bruker ER $4111 \mathrm{VT}$ variable temperature unit. Spectrometer settings were: applied microwave power $20 \mathrm{~mW}$, modulation frequency $100 \mathrm{kHz}$ and modulation amplitude $0.5 \mathrm{mT}$, appropriate to avoid saturation and /or over modulation of the Fe(III) signal. For simple comparison of signal strength all spectra were recorded with a sweep width $160 \mathrm{mT}, 1024$ points, time constant $10 \mathrm{~ms}$ with 20 sweeps added together for each measurement. Samples were analysed in an $8 \mathrm{~mm}$ tube (precision bore suprasil tube 513A-1PP-7SUP) fitted in a special made Dewar (WG-821-TMRSPECIAL) both from Wilmad-LabGlas (Vineland, NJ). No EPR signal could be detected for 
the empty sample tube or phosphate buffered saline (PBS). Plaque parts distal and proximal to the section used for histology (see section above) for each plaque were cut transversally in sections (30 mg - $70 \mathrm{mg}$ each). Each section was placed into a syringe and PBS was added to a total (sample + PBS) volume of $100 \mu \mathrm{L}$ to maintain the same sample volume. The syringe was frozen in liquid nitrogen, removed and warmed in the palms of the hands until a slightly thawed $100 \mu \mathrm{L}$ cylinder could be pressed out into the deep frozen sample tube, which was immediately placed in the EPR resonator. Signal intensity variation due to variations in microwave coupling and Q-value for all samples was minimised as the sample volumes were similar. The recorded EPR spectra were imported [35] into MATLAB (version R2011a, MathWorks, Inc.), baseline corrected and double integrated using an in-house developed MATLAB code. The EPR signal intensities were mass normalised. The quantity of Fe(III) in nmol Fe(III)/mg tissue could be calculated using calibration curves obtained from a series of repeated measurements on calibration samples (Fe(III)-deferoxamine 1:1 complex in PBS) in the range $0 \mathrm{mM}$ Fe(III) (PBS only) to $2.00 \mathrm{mM}$ Fe(III), typically in steps of $0.25 \mathrm{mM}$. The detection limit was approximately $0.01 \mathrm{nmol} \mathrm{Fe(III)/mg} \mathrm{sample.}$

\section{Statistical analysis}

All statistical analyses were performed using SPSS v.19 (IBM SPSS Statistics 19).

Descriptive statistics are presented as mean \pm standard deviation (SD) unless otherwise stated. Quantitative variables were analysed with independent samples Student t-tests and $p$ values $<0.05$ were considered statistically significant.

\section{Results}

Duplex ultrasound in vivo 
The degree of ICA stenosis measured as peak systolic flow velocities (PSV) showed no statistical significance between the groups $(4.9 \mathrm{~m} / \mathrm{s} \pm 1.8 \mathrm{~m} / \mathrm{s}$ (symptomatic) vs. $3.4 \mathrm{~m} / \mathrm{s} \pm 0.2$ $\mathrm{m} / \mathrm{s}$ (asymptomatic), $(\mathrm{P}>0.05))$. The degree of ICA stenosis in symptomatic patients were determined to be $80 \%-99 \%(n=6)$ and $70 \%-79 \%(n=1)$. The degree of ICA stenosis in asymptomatic patients were $80 \%$ - $99 \%(n=3)$. Contralateral significant ICA disease was seen in 3 symptomatic patients (occlusion, $\mathrm{n}=1$ and significant ICA-stenosis $50 \%-79 \%$, $\mathrm{n}$ $=2)$. The asymptomatic plaques were classified as fibrous $(n=2)$, fibro-atheromatous $(n=$ 1). The symptomatic plaques were classified as fibrous $(n=1)$, fibro-atheromatous $(n=2)$ and atheromatous $(n=4)$. In the symptomatic patient group all patients had signs of irregular cap surface combined with ulceration in two plaques. In the asymptomatic patient group two plaques had signs of smooth plaque surface and one had signs of irregular plaque surface. Plaque compositions and cap classifications are given in Table 1.

\section{Histopathology}

In all but one symptomatic plaques rupture could be identified while none of the asymptomatic plaques showed signs of rupture. The proportion of the cap that contained foamy macrophages in the symptomatic plaques was significantly higher compared to asymptomatic plaques $(31 \pm 11 \%$ vs $2.3 \pm 2.3, \mathrm{P}<0.01)$. A tendency of higher hemosiderin score could be seen in the symptomatic plaques but this did not reach statistical significance (results not shown). The presence of macrophages outside of the plaque, microvascular proliferation in the cap and in other parts of the section, and presence of calcification did not reach statistical significance between the groups. A typical example of an asymptomatic plaque with intact cap and few macrophages is shown in Figure 1, panel A. A symptomatic plaque with rupture and extensive invasion of foamy macrophages within the cap is shown in panel B. 


\section{EPR spectroscopy}

The EPR spectra of the slices from the 10 carotid atherosclerotic plaques consisted of haem iron signals, non-haem high-spin iron (e.g. iron in transferrin) and low-spin iron. A representative spectrum is shown in Figure 2 A with the low field signal from haem iron centred on $B_{0} \approx 1200 \mathrm{G}(\mathrm{g}=6)$, non-haem high-spin iron (e.g. iron bond in transferrin) centred on $B_{0} \approx 1600 \mathrm{G}(\mathrm{g}=4.3)$ and low-spin species centred on $B_{0} \approx 3400 \mathrm{G}(\mathrm{g}=2)$. Figure $2 \mathrm{~B}$ shows a magnification of the $\mathrm{g}=6$ and $\mathrm{g}=4.3$ signals ( $\mathrm{g}$ is a dimensionless quantity, which characterises the magnetic moment and gyromagnetic ratio of electrons and can be used to identify paramagnetic species in an EPR spectrum [32]).

\section{Quantification of Fe(III)}

Signal intensity analysis (of the peaks at $g=4.3$ ) showed large intra-plaque variations in $\mathrm{Fe}(\mathrm{III})$ concentrations with values ranging from below detection limit $(0.01 \mathrm{nmol} \mathrm{Fe}(\mathrm{III}) / \mathrm{mg}$ sample) up to $1.00 \mathrm{nmol} \mathrm{Fe}(\mathrm{III}) / \mathrm{mg}$ tissue within the same plaque. Data of all plaques are summarised in Table 1, Table 2, Table 3 and Figure 3. The mean Fe(III) concentration was significantly larger in symptomatic plaques $(0.29 \pm 0.08 \mathrm{nmol} \mathrm{Fe}(\mathrm{III}) / \mathrm{mg}$ sample $)$ as compared to asymptomatic plaques $(0.11 \pm 0.09 \mathrm{nmol} \mathrm{Fe}(\mathrm{III}) / \mathrm{mg}$ sample $)(\mathrm{P}<0.05)($ Table 2). When only considering the two to three sections adjoining the narrowest part of the plaque lumen, the difference was $(0.36 \pm 0.21 \mathrm{Fe}(\mathrm{III}) / \mathrm{mg}$ sample $)$ as compared to asymptomatic plaques $(0.06 \pm 0.04 \mathrm{Fe}(\mathrm{III}) / \mathrm{mg}$ sample $)(\mathrm{P}<0.05)$ (Table 2$)$. Table 1 shows the measured mean $\mathrm{Fe}(\mathrm{III})$ concentrations and maximum and minimum values for all plaques. Table 3 gives all the $\mathrm{Fe}(\mathrm{III})$ concentration in all sections for all plaques. Shaded areas and underlined numbers in Table 3 indicate sections adjoining the narrowest part of the plaques as determined with ex vivo ultrasound. The distribution of $\mathrm{Fe}(\mathrm{III})$ in one asymptomatic plaque 
(plaque \#1) and one symptomatic plaque (plaque \#10) is shown in Figure 4 and Figure 5 respectively. The dashed lines in Figure 4 and Figure 5 indicate the narrowest part for these plaques.

\section{Discussion}

These data support two main conclusions: (1) Symptomatic patients had significantly higher concentrations of $\mathrm{Fe}(\mathrm{III})$ as quantified by EPR spectroscopy, as well as signs of cap rupture and increased cap macrophage activity, as compared to asymptomatic patients. (2) The intraplaque distribution of $\mathrm{Fe}(\mathrm{III})$ in ex vivo atherosclerotic plaques is heterogeneous.

Intra-plaque haemorrhage and presence of neovascularization and have been correlated with the incidence of earlier cardiovascular events [36-37]. Visualization of neovascularization and haemorrhage can be performed noninvasively using imaging modalities such as contrastenhanced ultrasound and MRI [38]. In this study we performed an ex vivo analysis using electron paramagnetic resonance (EPR) spectroscopy to assess data concerning differences in iron content as a sign of haemorrhage in clinical silent plaques as compared to plaques provoking neurological symptoms.

Stadler et al. [16] used both EPR spectroscopy and inductively coupled plasma mass spectroscopy (ICP-MS) to quantify iron in ex vivo carotid plaques. They found that carotid plaques that had been classified as "complex" or "calcified" contained statistically higher levels of $\mathrm{Fe}$ (III) as compared to healthy intima samples or plaques classified as "clean" and concluded that elevated levels of Fe(III) may be related to plaque instability. Raman et al. [19] found that $\mathrm{T}_{2}{ }^{*}$ (an MRI relaxation parameter measured in ms) was significantly shorter in symptomatic patients as compared to asymptomatic patients, which indicated higher amounts of $\mathrm{T}_{2}{ }^{*}$ shortening by iron aggregates in symptom producing atherosclerotic plaques. 
However, Raman et al. [19] found, contradictory to Stadler et al. [16], lower levels of Fe(III) in plaques from symptomatic patients, as compared to asymptomatic patients.

In this study we included plaques both from symptomatic patients referred to ultrasound examination because of neurological symptoms (TIA, minor stroke and amaurosis fugax) as well as asymptomatic patients (i.e. carotid plaques without neurological symptoms).

Various techniques such as ultrasound, magnetic resonance imaging (MRI), dual energy CT (DECT) and positron emission tomography (PET) are available for visualization of carotid plaques. MRI has been shown to be useful for identification of risk factors for rupture such as vascular wall inflammation, fibrous cap, lipid rich necrotic core and neovascularization [2930]. However, despite recent developments of conventional imaging modalities it is still a clinical challenge to predict the vulnerability for plaque rupture and subsequent embolic stroke [14].

Oxidative stress is a causative factor for the pathogenesis of vascular disease [9] and iron may act as a catalyst for the generation of ROS during its cycling between oxidation states $\mathrm{Fe}(\mathrm{III})$ and $\mathrm{Fe}(\mathrm{II})$ by means of Fenton and Haber-Weiss reactions [24, 32]. Iron could therefore be central in the pathogenesis of atherosclerosis. It has even been postulated that the gender differences seen in death from heart disease depends on the gender differences in body iron stores $[15,20]$.

Almost all iron in the body is tightly bound by proteins in redox inactive forms such as serum ferritin $[18,21]$ and can thus not act as a catalyst for ROS. However, it is known that iron can be released from ferritin by several pathological processes [25] and thus ferritin-dependent oxidative damage can be induced by pathological leakage of iron from e.g. ferritin to form "catalytic" or "labile" iron [23], in which the iron is bound by low molecular weight ligands 
like organic acids and nucleotides [18].

All our ten carotid atherosclerotic plaques gave EPR spectra that to various degrees consisted of haem iron signals, non-haem high-spin iron (e.g. iron in transferrin) and low-spin iron (Figure 2). We choose to quantify the amount of non-haem high-spin iron (e.g. iron in transferrin) in sections of each plaque using quantitative EPR spectroscopy at $150 \mathrm{~K}$ using signal intensity comparisons with calibration samples (Fe(III)-deferoxamine 1:1 complex in PBS) with known concentrations. Our method for quantitative EPR of Fe(III) with measurements on frozen $100 \mu \mathrm{L}$ discs in a $8 \mathrm{~mm}$ tube fitted in a special made Dewar allowed for high measurement accuracy and precision as estimated from a series of repeated measurements on both calibration samples and carotid plaques. Our results (Table 2 and Figure 3) are in agreement of the findings by Stadler et al. [16] with higher mean non-haem high-spin iron $(g=4.3)$ concentrations in atherosclerotic plaques from symptomatic patients. We also found that the intra-plaque distributions of non-haem high-spin iron (e.g. iron in transferrin) are very heterogeneously distributed in carotid atherosclerotic plaques as exemplified by Figure 4 and Figure 5.

The histopathological examination revealed that the symptomatic plaques had significant higher presence of macrophages and presence of rupture in all but one case. These differences confirmed the symptomatic plaques as vulnerable. Other classical signs of vulnerable plaques such as larger lipid core, thinner fibrous cap or microvascular proliferation [39] were not found to differ in the present material. Nevertheless the sign of cap rupture in combination with increased macrophage activity that we found in this study could be a critical sign of vulnerability. There could be several reasons for this discrepancy of vulnerability signs and the main one being the small number of cases in the present study. We 
must also consider the possibility that differences exist between the mostly described coronary vessel plaques and those in the carotids. It can be argued that an increase in iron could be secondary to neovascularization, but the histopathological examination did not support this assumption. However, a tendency to higher hemosiderin content in the symptomatic, vulnerable plaques was observed. This could be an indirect sign of increased leakiness in less well endothelialized vessels as present during neovascularization.

Whether differences in plaque structure alone are enough to distinguish between symptomatic and clinically silent lesions has yet to be established [34]. It has been reported that ultrasonic echolucent carotid plaques predict future strokes [40], but the cap surface might be the best ultrasound predictor to assess the vulnerability of the plaque as shown to be ruptured in the symptomatic plaques.

Obviously, as expected, other risk factors, in addition to the peak value of $\mathrm{Fe}(\mathrm{III})$ concentration, are important for plaque vulnerability for rupture. Our hypothesis that vulnerability for rupture of carotid atherosclerotic plaques is correlated to intra-plaque iron distribution is to some extent but not fully supported by the tendency of larger difference in mean iron concentration between the groups when only considering the position determined as rupture prone (most narrow) using ultrasound (Table 2). While the number of plaques ( $\mathrm{n}=$ 10) has to be increased in further studies, the results indicate that the distribution of iron in atherosclerotic plaques can be associated with local vulnerability for rupture. We believe that dedicated MRI studies are needed in the future to establish if the mean iron concentration alone can be used to predict plaque vulnerability for rupture, or if the mean iron concentration rather should be evaluated together with MRI observations of other risk factors such as lipid rich necrotic core, vascular wall inflammation et cetera. Our observation that the Fe(III) 
distribution varies substantially within atherosclerotic plaques could possible point towards that the latter combined approach is needed. Fe(III) concentrations up to $0.92 \mathrm{nmol}$ $\mathrm{Fe}(\mathrm{III}) / \mathrm{mg}$ tissue in one tissue section were observed in one plaque from an asymptomatic patients (Table 3 , section 6 of plaque \#3). Interestingly ultrasound showed signs of irregular cap surface in this plaque indicating high risk of embolisation compared to the other two asymptomatic plaques with signs of smooth cap. Plaque \#3 therefore mimicked the symptomatic plaques concerning cap surface whereas all symptomatic plaques had signs of irregular cap and / or ulceration. The vulnerability of the plaque is especially important to assess in asymptomatic patients before the onset of neurological symptoms.

One could argue that the highest risk of embolisation is supposed to occur when the cap of the plaque is broken due to high concentration of macrophages in the cap. We therefore included macrophage activity as well as description of the cap in the first place in this study and did not include oxidative epitopes which however could be an important topic for further studies.

Conclusion: The results of this study indicate that Fe(III) distribution varies substantially within atherosclerotic plaques. Plaques from symptomatic patients had significantly higher concentrations of Fe(III), as well as signs of cap rupture and increased cap macrophage activity.

\section{Acknowledgments}

H. Gustafsson acknowledges The Swedish Research Council (diarienr 2009-5430) for financial support and Stiftelsen Lars Hiertas Minne for travel grant (FO2009-0345). M. Engström acknowledges the Medical Research Council of Southeast Sweden (FORSS) for 
financial support. M. Lindgren thanks Linköping University for the tenure as distinguished guest professor. We thank B. Frånlund and L. Palmqvist for histopathological preparations.

\section{References}

[1] Virmani R, Burke AP, Farb A, Kolodgie FD. Pathology of the vulnerable plaque. J. Am. Coll. Cardiol. 2006; 47: C13 - C18.

[2]

Libby P. Inflammation in atherosclerosis. Nature. 2002; 420: 868 - 874.

[3] Hansson GK . Inflammation, Atherosclerosis, and Coronary Artery Disease. N. Engl. J. Med. 2005; 352: 1685 - 1695.

[4] Finn AV, Nakano M, Narula J, Kolodgie FD, Virmani R. Concept of Vulnerable/Unstable Plaque. Arterioscler. Thromb. Vasc. Biol. 2010; 30: 12821292.

[5]

Lendon CL, Davies MJ, Born GVR, Richardson PD. Atherosclerotic plaque caps are locally weakened when macrophages density is increased. Atherosclerosis. 1991; 87: $87-90$.

[6] Stocker R, Keaney Jr JF. Role of Oxidative Modifications in Atherosclerosis. Physiol. Rev. 2004; 84: 1381 - 1478.

[7] Channon KM. Oxidative Stress and Coronary Plaque Stability. Arterioscler. Thromb. Vasc. Biol. 2002; 22: 1751-1752. 
Disease. Arterioscler. Thromb. Vasc. Biol. 2005; 25: 29 - 38.

[9] Drummond GR, Selemidis S, Griendling KK, Sobey CG. Combating oxidative stress in vascular disease: NADPH oxidases as therapeutic targets. Nat. Rev. Drug Discov. 2011; 10: 453-471.

[10] Rajagopalan S, Meng XP, Ramasamy S, Harrison DG, Galis ZS. Reactive oxygen species produced by macrophage-derived foam cells regulate the activity of vascular matrix metalloproteinases in vitro. Implications for atherosclerotic plaque stability. J. Clin. Invest. 1996; 98: 2572-2579.

[11] Barnett HJ, Taylor DW, Eliasziw M, Fox AJ, Ferguson GG, Haynes RB, Rankin RN, Clagett GP, Hachinski VC, Sackett DL, Thorpe KE, Meldrum HE, Spence JD. Benefit of carotid endarterectomy in patients with symptomatic moderate or severe stenosis: North American Symptomatic Carotid Endarterectomy Trial Collaborators. N. Engl. J. Med. 1998; 339: 1415-1425.

[12] Asymptomatic Carotid Atherosclerosis Study Group. Carotid endarterectomy for patients with asymptomatic internal carotid artery stenosis. JAMA 1995; 273: $1421-28$.

[13] Halliday A, Harrison M, Hayter E, Kong X, Mansfield A, Marro J, Pan H, Peto R, Potter J, Rahimi K, Rau A, Robertson S, Streifler J, Thomas D. 10-year 
stroke prevention after successful carotid endarterectomy for asymptomatic stenosis (ACST-1): a multicentre randomised trial. Lancet 2010; 376: 10741084.

[14] Finn AV, Kolodgie FD, Virmani R. Correlation between carotid intimal/medial thickness and atherosclerosis: a point of view from pathology. Arterioscler. Thromb. Vasc. Biol. 2010; 30: 177-181.

[15] Sullivan JL. Iron and the sex difference in heart disease risk. Lancet. 1981; 317: $1293-1294$.

[16] Stadler N, Lindner RA, Davies MJ. Direct detection and quantification of transition metal ions in human atherosclerotic plaques: evidence for the presence of elevated levels of iron and copper. Arterioscler. Thromb. Vasc. Biol. 2004; 24: 949 - 954.

[17] Stanley N, Stadler N, Woods AA, Bannon PG, Davies MJ. Concentrations of iron correlate with the extent of protein, but not lipid, oxidation in advanced human atherosclerotic lesions. Free Radic. Biol. Med. 2006; 40: 1636 - 1643.

[18] Lapenna D, Pierdomenico SD, Ciofani G, Ucchino S, Neri M, Giamberardino MA, Cuccurullo F. Association of body iron stores with low molecular weight iron and oxidant damage of human atherosclerotic plaques. Free Radic. Biol. Med. 2007; 42: $492-498$. 
[19] Raman SV, Winner III MW, Tran T, Velayutham M, Simonetti OP, Baker PB, Olesik J, McCarthy B, Ferketich AK, Zweier JL. In Vivo Atherosclerotic Plaque Characterization Using Magnetic Susceptibility Distinguishes SymptomProducing Plaques. J. Am. Coll. Cardiol. Img. 2008; 1: 49 - 57.

[20] Sullivan JL. Iron in arterial plaque: A modifiable risk factor for atherosclerosis. Biochim. Biophys. Acta. 2009; 1790: 718-723.

[21] Formanowicz D. Do changes in iron metabolism contribute to the acceleration of the atherosclerosis process? Journal of Biotechnology, Computational Biology and Bionanotechnology. 2011; 2: 180-192.

[22] Lioupis C, Barbatis C, Drougou A, Koliaraki V, Mamalaki A, Klonaris C, Georgopoulos S, Andrikopoulos V, Bastounis E. Association of haptoglobin genotype and common cardiovascular risk factors with the amount of iron in atherosclerotic carotid plaques. Atherosclerosis. 2011; 216: $131-138$.

[23] Rajapurkar MM, Shah SV, Lele SS, Hegde UN, Lensing SY, Gohel K, Mukhopadhyay B, Gang S, Eigenbrodt ML. Association of catalytic iron with cardiovascular disease. Am. J. Cardiol. 2012; 109: 438 - 442.

[24] Wardman P, Candeias LP. Fenton Chemistry: An Introduction. Radiat. Res. 1996; 145: 523-531. 
[25] Reif DW. Ferritin as a source of iron for oxidative damage. Free Radic. Biol. Med. 1992; 12: 417 - 427.

[26] Doyle FH, Pennock JM, Banks LM, McDonnell MJ, Bydder GM, Steiner AE, Young IR, Clarke GJ, Pasmore T, Gilderdale DJ. Nuclear Magnetic Resonance Imaging of the Liver: Initial Experience. Am. J. Roentgenol. 1982; 138: 193200.

[27] Stark DD, Moseley ME, Bacon BR, Moss AA, Goldberg HI, Bass NM, James TL. Magnetic resonance imaging and spectroscopy of hepatic iron overload. Radiology. 1985; 154: 137-142.

[28] Anderson LJ. Assessment of Iron Overload with T2* Magnetic Resonance Imaging. Prog. Cardiovasc. Dis. 2011; 54: 287-294. Takaya N, Yuan C, Chu B, Saam T, Underhill H, Cai J, Tran N, Polissar NL, Isaac C, Ferguson MS, Garden GA, Cramer SC, Maravilla KR, Hashimoto B, Hatsukami TS. Association between carotid plaque characteristics and subsequent ischemic cerebrovascular events: a prospective assessment with MRI - initial results. Stroke. 2006; 37: 818 - 823.

[30] Hatsukami T, Yuan C. MRI in the early identification and classification of highrisk atherosclerotic plaques. Imaging Med. 2010; 1: 63-75. 
[31] Crichton R. Iron metabolism. From molecular mechanisms to clinical consequences. Singapore: John Wiley \& Sons Ltd; 2009.

[32] Symons MCR, Gutteridge JMC. Free radicals and iron: chemistry, biology, and medicine. Oxford: Oxford University Press; 2002.

[33] Pilbrow JR. Transition ion electron paramagnetic resonance. Oxford: Oxford University Press; 1990.

[34] Jogestrand T, Eiken O, Nowak J. Relation between the elastic properties and intima-media thickness of the common carotid artery. Clin. Physiol. Funct. Imaging. 2003; 23: 134-137.

Stoll S, Schweiger A. EasySpin, a comprehensive software package for spectral simulation and analysis in EPR. J. Magn. Reson. 2006; 178: 42-55. Carotid Plaque Vulnerability: Impact on Ischemic Stroke. Arch. Immunol. Ther. Exp. 2012; 60: 431-442. Martin-Ventura JL. From intraplaque haemorrhages to plaque vulnerability: biological consequences of intraplaque haemorrhages. J. Cardiovasc. Med. 2012; 13: 628-634. 

and hemorrhage: markers for cardiovascular risk stratification and therapeutic monitoring. J. Cardiovasc. Med. 2012; 13: 635-639. atherosclerotic plaque: scope of the literature. Ann. Intern. Med. 2010; 153: $387-395$

[40] Grønholdt M-LM, Nordestgaard BG, Schroeder TV, Vorstrup S, Sillesen H. Ultrasonic Echolucent Carotid Plaques Predict Future Strokes. Circulation. 2001; 104: 68-73. 


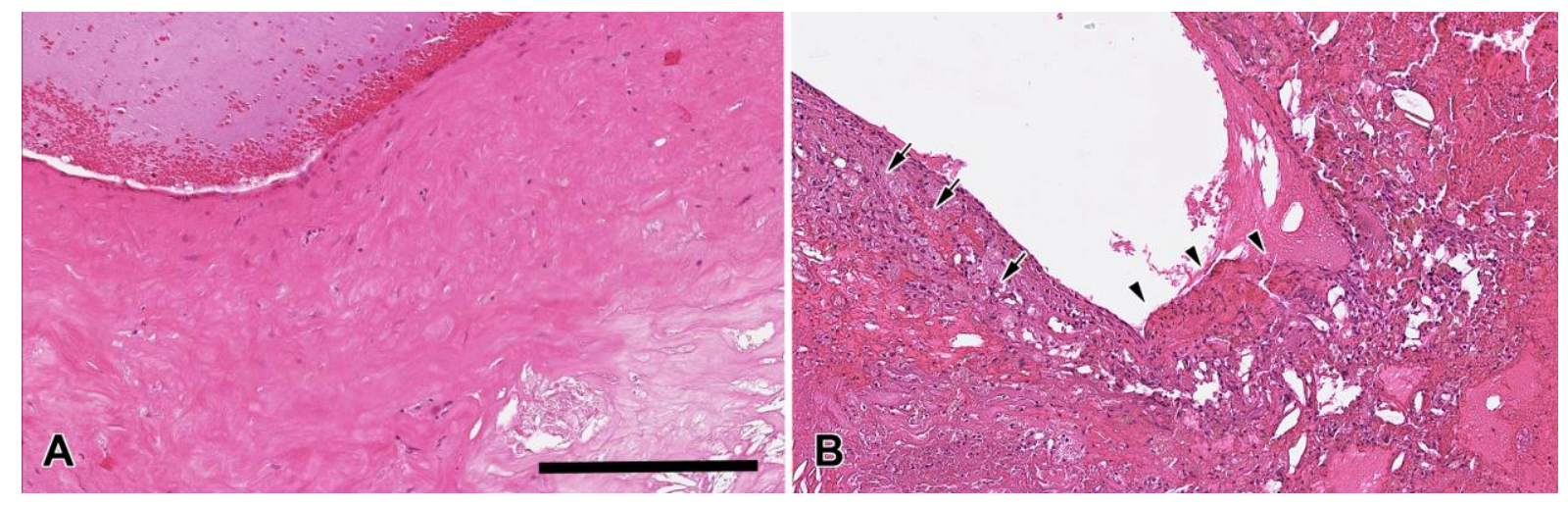

Figure 1. Panel A shows a typical example of an asymptomatic plaque with intact cap and little macrophages. Panel B shows a symptomatic plaque with rupture (arrowheads) and extensive invasion of foamy macrophages (arrows) within the cap. Scale bar $=200 \mathrm{um}$.
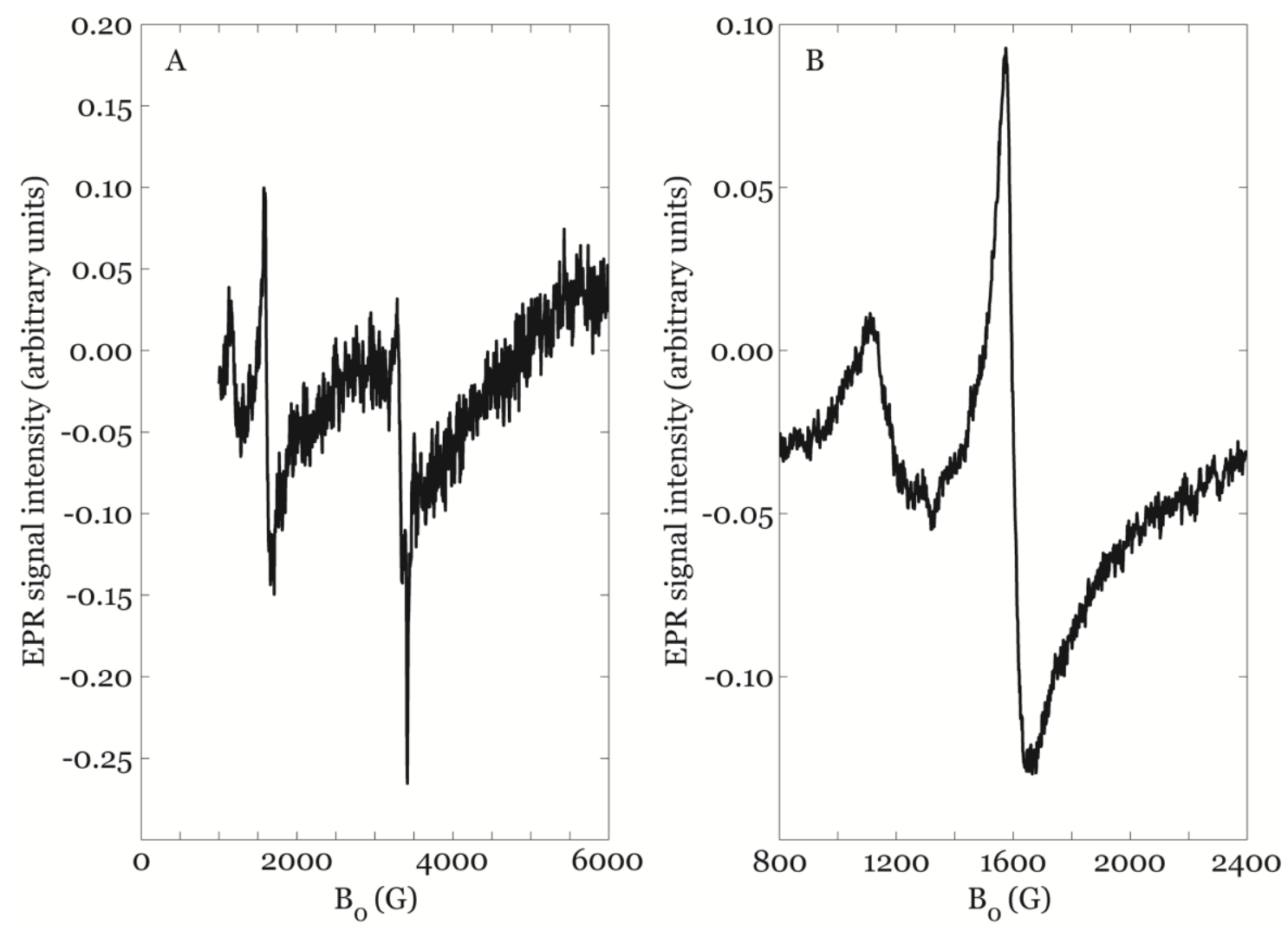

Figure 2. Panel A shows a representative EPR spectrum from a frozen human carotid atherosclerotic plaque (one section obtained from plaque \# 4, symptomatic patient) with a low field signal from haem iron centred on $B_{0} \approx 1200 \mathrm{G}(\mathrm{g}=6)$, non-haem high-spin iron (e.g. iron bond in transferrin) centred on $B_{0} \approx 1600 \mathrm{G}(\mathrm{g}=4.3)$ and low-spin species centred on $B_{0} \approx 3400 \mathrm{G}(\mathrm{g}=2)$. Panel B shows a magnification of the $\mathrm{g}=6$ and $\mathrm{g}=4.3$ signals. 


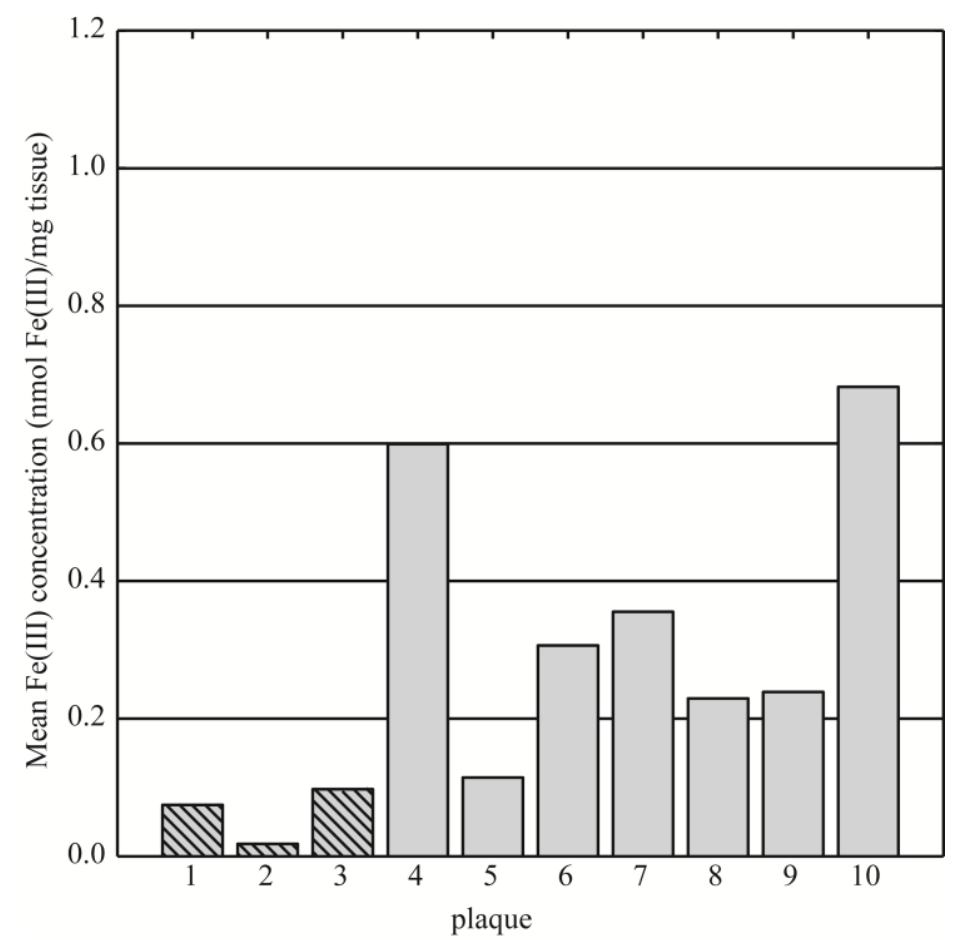

Figure 3. Mean Fe(III) concentration in the two - three sections adjoining the narrowest part of the lumen (nmol Fe(III) /mg tissue). Plaques 1 - 3 from asymptomatic patients. Plaques 4 10 from symptomatic patients. Significantly higher mean Fe(III) in sections from symptomatic patients as compared to sections from asymptomatic patients $(\mathrm{P}<0.05)$.

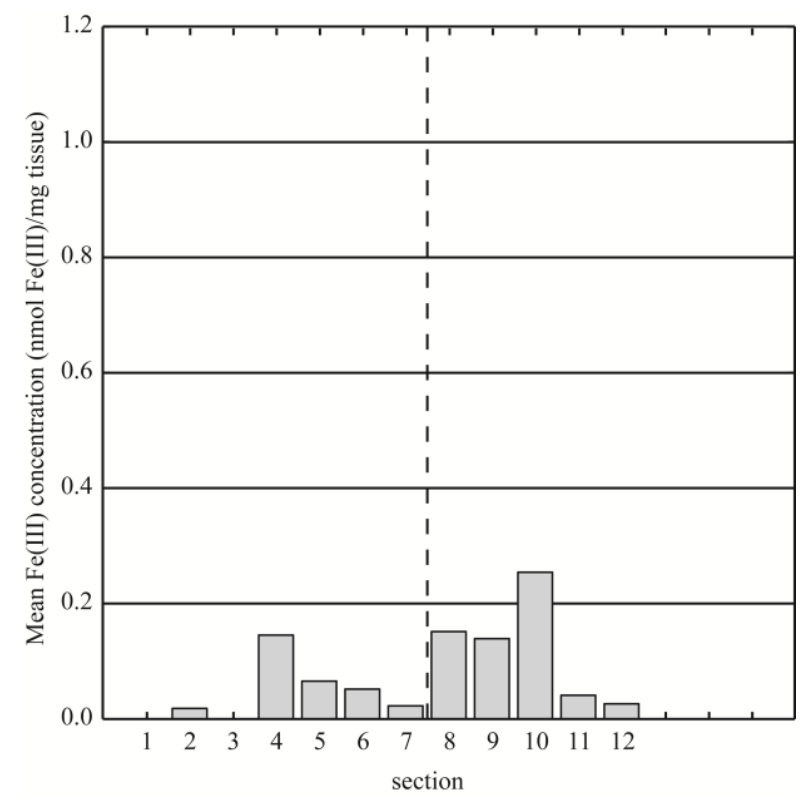

Figure 4. Experimental results from EPR spectroscopy of asymptomatic plaque number 1.

Dashed line indicates the narrowest part of the lumen as indicated by ex vivo ultrasound. 


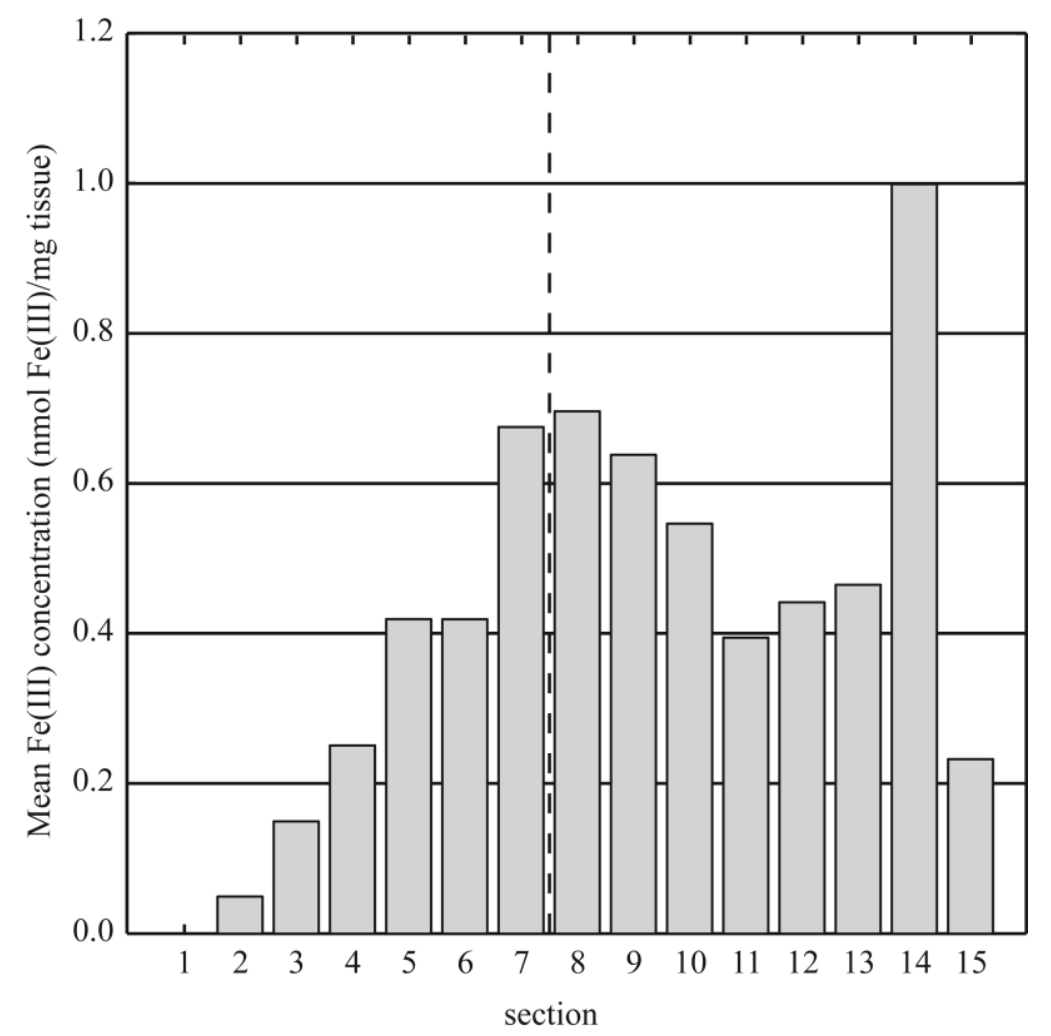

Figure 5. Experimental results from EPR spectroscopy of symptomatic plaque number 10.

Dashed line indicates the narrowest part of the lumen as indicated by ex vivo ultrasound. 
Table 1. Clinical classification, plaque composition, cap surface and mean, maximum and minimum Fe(III) concentration for all plaques.

\begin{tabular}{|c|c|c|c|c|c|c|c|}
\hline plaque \# & clinical classification & $\begin{array}{l}\text { Plaque composition } \\
\text { (fibrous, fibro- } \\
\text { atheromatous or } \\
\text { atheromatous) }\end{array}$ & $\begin{array}{c}\text { cap surface } \\
\text { (smooth, irregular or } \\
\text { ulcerated) }\end{array}$ & $\begin{array}{l}\text { mean Fe(III) } \\
\text { concentration in all } \\
\text { tissue sections } \\
(\text { nmol Fe(III) } \\
/ \text { mg tissue) }\end{array}$ & $\begin{array}{c}\text { mean Fe(III) } \\
\text { concentration in } \\
\text { tissue sections } \\
\text { adjoining } \\
\text { narrowest part } \\
\text { (nmol Fe(III) } \\
\text { /mg tissue) } \\
\end{array}$ & $\begin{array}{l}\text { Maximum intra- } \\
\text { plaque Fe(III) } \\
\text { concentration } \\
\text { (nmol Fe(III) } \\
\text { /mg tissue) }\end{array}$ & $\begin{array}{l}\text { Minimum intra- } \\
\text { plaque Fe(III) } \\
\text { concentration } \\
\text { (nmol Fe(III) } \\
\text { /mg tissue) }\end{array}$ \\
\hline$\# 1$ & asymptomatic & fibrous & smooth & 0.08 & 0.07 & 0.25 & $<0.01$ \\
\hline$\# 2$ & asymptomatic & fibro-atheromatous & smooth & 0.03 & 0.02 & 0.09 & $<0.01$ \\
\hline$\# 3$ & asymptomatic & fibrous & irregular & 0.21 & 0.10 & 0.92 & $<0.01$ \\
\hline$\# 4$ & symptomatic & fibro-atheromatous & irregular & 0.34 & 0.60 & 0.62 & $<0.01$ \\
\hline$\# 5$ & symptomatic & fibrous & irregular & 0.15 & 0.12 & 0.37 & $<0.01$ \\
\hline$\# 6$ & symptomatic & atheromatous & irregular & 0.26 & 0.31 & 0.38 & 0.16 \\
\hline$\# 7$ & symptomatic & fibro-atheromatous & ulcerated & 0.27 & 0.36 & 0.54 & 0.11 \\
\hline$\# 8$ & symptomatic & atheromatous & irregular & 0.28 & 0.23 & 0.76 & 0.04 \\
\hline$\# 9$ & symptomatic & atheromatous & irregular & 0.31 & 0.24 & 0.70 & $<0.01$ \\
\hline$\# 10$ & symptomatic & atheromatous & ulcerated & 0.43 & 0.69 & 1.00 & $<0.01$ \\
\hline
\end{tabular}


Table 2.Mean Fe(III) concentration for all plaques from symptomatic $(\mathrm{n}=7)$ and asymptomatic patients $(\mathrm{n}=3)$. Values are given as mean value \pm standard deviation $(\mathrm{k}=1)$.

\section{adjoining narrowest part}

(nmol Fe(III)

/mg tissue) (nmol Fe(III)

/mg tissue)

\begin{tabular}{llr}
\hline asymptomatic & $0.11 \pm 0.09$ & $0.06 \pm 0.04$ \\
symptomatic & $0.29 \pm 0.08^{*}$ & $0.36 \pm 0.21^{*}$ \\
\end{tabular}

${ }^{*}$ Significantly higher versus plaques from asymptomatic patients $(\mathrm{P}<0.05)$. 
Table 3. Fe(III) in all tissue sections for all plaques. Shaded areas indicate tissue sections adjoining narrowest part of the plaques as determined with ex vivo ultrasound.

\begin{tabular}{|c|c|c|c|c|c|c|c|c|c|c|}
\hline $\begin{array}{c}\mathrm{nmol} \mathrm{Fe}(\mathrm{III}) \\
\text { /mg tissue }\end{array}$ & plaque \#1 & plaque \#2 & plaque \#3 & plaque \#4 & plaque $\# 5$ & plaque \#6 & plaque \#7 & plaque \#8 & plaque \#9 & plaque $\# 10$ \\
\hline section 1 & $<0.01$ & $<0.01$ & 0.50 & $<0.01$ & 0.07 & 0.27 & 0.13 & $\underline{0.06}$ & $<0.01$ & $<0.01$ \\
\hline section 2 & 0.02 & 0.09 & $\underline{0.17}$ & 0.15 & $\overline{0.16}$ & 0.16 & 0.22 & $\overline{0.41}$ & $<0.01$ & 0.05 \\
\hline section 3 & $<0.01$ & $\underline{0.04}$ & $\underline{0.02}$ & $\underline{0.59}$ & $\overline{0.15}$ & 0.26 & 0.31 & $\overline{0.76}$ & 0.29 & 0.15 \\
\hline section 4 & 0.15 & $\leq 0.01$ & $<0.01$ & 0.62 & $<0.01$ & 0.24 & 0.11 & 0.64 & 0.39 & 0.25 \\
\hline section 5 & 0.07 & $\overline{0.06}$ & 0.17 & $\overline{0.19}$ & 0.37 & $\overline{0.38}$ & $\underline{0.18}$ & 0.14 & 0.30 & 0.42 \\
\hline section 6 & $\underline{0.05}$ & $<0.01$ & 0.92 & 0.27 & 0.28 & & $\overline{0.54}$ & 0.13 & 0.24 & 0.42 \\
\hline section 7 & $\overline{0.02}$ & $<0.01$ & $<0.01$ & 0.35 & $<0.01$ & & $\overline{0.42}$ & 0.14 & $\leq 0.01$ & $\underline{0.68}$ \\
\hline section 8 & $\underline{0.15}$ & 0.03 & 0.11 & 0.53 & & & & 0.17 & $\underline{0.48}$ & $\overline{0.70}$ \\
\hline section 9 & $\overline{0.14}$ & & 0.12 & & & & & 0.27 & $\overline{0.70}$ & $\overline{0.64}$ \\
\hline section 10 & 0.25 & & 0.11 & & & & & 0.04 & 0.67 & 0.55 \\
\hline section 11 & 0.04 & & & & & & & & & 0.39 \\
\hline section 12 & 0.03 & & & & & & & & & 0.44 \\
\hline section 13 & & & & & & & & & & 0.46 \\
\hline section 14 & & & & & & & & & & 1.00 \\
\hline section 15 & & & & & & & & & & 0.23 \\
\hline
\end{tabular}


